\title{
Pricing American contingent claims by stochastic linear programming
}

\section{Ahmet Camcı \& Mustafa Ç. Pınar}

To cite this article: Ahmet Camcı \& Mustafa Ç. Pınar (2009) Pricing American contingent claims by stochastic linear programming, Optimization, 58:6, 627-640, DOI: 10.1080/02331930902819188

To link to this article: http://dx.doi.org/10.1080/02331930902819188

Published online: 22 Jul 2009.

\section{Submit your article to this journal ¿}

III Article views: 69

View related articles $\asymp$

7 Citing articles: 9 View citing articles 진 


\title{
Pricing American contingent claims by stochastic linear programming
}

\author{
Ahmet Camcı* and Mustafa Ç. Pınar \\ Department of Industrial Engineering, Bilkent University, 06800 Bilkent, Ankara, Turkey
}

(Received 25 July 2007; final version received 18 August 2008)

\begin{abstract}
We consider pricing of American contingent claims (ACC) as well as their special cases, in a multi-period, discrete time, discrete state space setting. Until now, determining the buyer's price for ACCs required solving an integer programme unlike European contingent claims for which solving a linear programme is sufficient. However, we show that a relaxation of the integer programming problem that is a linear programme, can be used to get the same lower bound for the price of the ACC.
\end{abstract}

Keywords: American contingent claim; pricing; hedging; martingales; stochastic linear programming

AMS Subject Classifications: 90C11; 90C90; 91B28

\section{Introduction}

Mathematical programming tools, especially stochastic programming (see [12] for a recent survey) are becoming increasingly useful as an entry point to studying the specialized methods of mathematical finance $[5,8,9]$. In this note, we are interested in the pricing of American Contingent Claims (ACC) as well as their special cases, in a multi-period, discrete time, discrete state space framework.

In the area of pricing contingent claims research concentrates mainly on defining and characterizing the range of contingent claim prices consistent with the absence of arbitrage. This range is determined by the upper hedging and the lower hedging prices, also known as the superreplication and subreplication bounds. In the absence of arbitrage, the upper hedging price is the value of the least costly self-financing portfolio strategy composed of market instruments whose pay-off is at least as large as the contingent claim pay-off. This price can also be interpreted from the perspective of a writer (seller) of the contingent claim as the smallest initial wealth required to replicate the contingent claim pay-off at expiration in a self-financed manner. Hence, we refer to the upper hedging price as the writer's price as well. Similarly, the lower hedging price is the value of the most precious self-financing portfolio strategy composed of market instruments whose pay-off is dominated by

*Corresponding author. Email: camci@bilkent.edu.tr 
the contingent claim pay-off at expiration. The lower hedging price can also be interpreted as the largest amount the contingent claim buyer can borrow (in the form of cash or by short-selling stocks) to acquire the claim while paying off his/her debt in a self-financed manner using the contingent claim pay-off at expiration [3]. Hence, we refer to this price as the buyer's price as well as the lower hedging price. For European contingent claims (ECC), which can only be exercised at expiration, the upper and lower hedging prices are usually expressed as supremum and infimum, respectively, of the expectation of the discounted contingent claim pay-off (at expiration) over all probability measures that make the underlying stock price a martingale. We direct the reader to the book by Föllmer and Schied [6] for an in-depth treatment of pricing contingent claims in discrete time.

Similar expectation expressions were developed by Harrison and Kreps [7] and Chalasani and Jha [3] for ACC, which can be exercised at any time until expiration. However, the possibility of early exercise complicates the expressions where one has to take supremums over all stopping times which represent potential exercise strategies of the contingent claim buyer. In particular, the upper hedging price is the supremum of the expectation of the discounted contingent claim pay-off (at some time between now and expiration) over all stopping times and all probability measures that make the underlying stock-price process a martingale. While the upper hedging price can be cast as a linear programming problem in discrete time [3,9], the lower hedging price is harder to compute. It is the supremum over all stopping times of the infimum of the expected discounted contingent claim pay-off (at some time between now and expiration) over all probability measures that make the underlying stock price process a martingale. More precisely, the lower hedging price of an ACC is given by an expression of the form

$$
\max _{\tau \in \mathcal{T}} \min _{P \in \mathcal{P}} \mathbb{E}^{P}\left[F_{\tau}\right]
$$

where $\mathcal{T}$ is the set of stopping times, $\mathcal{P}$ is the set of all martingale measures, and $F_{\tau}$ is the discounted contingent claim pay-off at time $\tau$; see e.g. Theorem 12.4 of [3].

Against this background, Pennanen and King [9] showed that the above expression for the lower hedging price can also be cast as

$$
\min _{P \in \mathcal{P}} \max _{\tau \in \mathcal{T}} \mathbb{E}^{P}\left[F_{\tau}\right]
$$

by interchanging the order of the max and min after observing that the outer maximization over the set $\mathcal{T}$ of stopping times can be replaced by maximization over a set of randomized stopping times, a central notion in [3] (see also the definition of the sets $E$ and $\tilde{E}$ just before Theorem 2 in the present paper) and convex duality theory. From an optimization point of view, Pennanen and King's characterization of the set of the lower hedging price for ACCs follows from a representation of the buyer's price as the optimal value of a linear programming problem in the hedging space of the buyer, instead of posing the same hedging problem over integer valued variables. This important observation opens the way to harnessing the welldeveloped linear programming algorithms and software for the calculation of the buyer's price for ACCs. However, while their result is correct, their proof has a serious gap that we shall explain in Section 3 through a counterexample. In this note we present an alternative proof of this result. After defining the buyer's problem 
similarly to the one in [9] we formulate an integer programming problem for the buyer's price. Then, we prove that the bound from the buyer's perspective can be computed by solving a linear programme. This result gives a correct alternative proof of Theorem 3 of [9]. Independently, Flåm [5] proves a similar result for the contingent claim writer's price using considerations of total unimodularity. However, as discussed above the computation of the lower and upper hedging prices leads to different problems where it appears that the buyer's problem is harder to analyse. In fact, Pennanen and King [9] also give an analysis of the writer's pricing problem. Hence, we concentrate on the buyer's problem in this note. Our proof uses direct construction of an integral optimal solution from a fractional solution. The result remains valid for dividend paying stocks as well. The significance of the result stems from the fact that there exist linear programming algorithms with a computational complexity bounded above by a low-order polynomial in the number of variables and constraints for computing a solution to $\epsilon$-accuracy; see Chapter 6 of [1]. In practice, one has access to numerous software packages capable of handling very large instances of linear programmes with dimensions reaching hundred thousand variables and constraints. Based on our experiences with European index options [10], multi-period hedging problems with approximately 70,000 variables and 22,000 equality, and 40,000 inequality constraints can be solved very quickly using the GAMS/CPLEX solver [2,4].

\section{The stochastic scenario tree and ACC}

An ACC $F$ is a financial instrument generating a real-valued stochastic (cash-flow) process $\left(F_{t}\right)_{t=0, \ldots, T}$. At any stage $t=0, \ldots, T$, the holder of an ACC may decide to take $F_{t}$ in cash and terminate the process. Using this definition, an American call option on a stock $S$ with strike price $K$ corresponds to $F=S-K$. American put is obtained by reversing the sign of $F$. We can define a European call option with maturity $T$ by setting $F_{t}=0$ for $t \neq T$. Bermudan call options having exercise date set $G \subset\{1, \ldots, T\}$ can be defined by setting $F_{t}=0$ for $t \notin G$.

To lay down a pricing framework based on no-arbitrage arguments for contingent claims, we assume that security prices and other payments are discrete random variables supported on a finite probability space $(\Omega, \mathcal{F}, P)$ whose atoms are sequences of real-valued vectors (asset values) over discrete time periods $t=0,1, \ldots, T$. We further assume that the market evolves as a discrete, nonrecombinant scenario tree (hence, suitable for incomplete markets) in which the partition of probability atoms $\omega \in \Omega$ generated by matching path histories up to time $t$ corresponds one-to-one with nodes $n \in \mathcal{N}_{t}$ at level $t$ in the tree. The set $\mathcal{N}_{0}$ consists of the root node $n=0$, and the leaf nodes $n \in \mathcal{N}_{T}$ correspond one-to-one with the probability atoms $\omega \in \Omega$. The $\sigma$-algebras are such that, $\mathcal{F}_{0}=\{\emptyset, \Omega\}, \mathcal{F}_{t} \subset \mathcal{F}_{t+1}$ for all $0 \leq t \leq T-1$ and $\mathcal{F}_{T}=\mathcal{F}$. A stochastic process is said to be $\left(\mathcal{F}_{t}\right)_{t=0}^{T}$-adapted if for each $t=0, \ldots, T$, the outcome of the process only depends on the element of $\mathcal{F}_{t}$ that has been realized at stage $t$. Similarly, a decision process is said to be $\left(\mathcal{F}_{t}\right)_{t=0}^{T}$-adapted if for each $t=0, \ldots, T$, the decision depends on the element of $\mathcal{F}_{t}$ that has been realized at stage $t$. In the scenario tree, every node $n \in \mathcal{N}_{t}$ for $t=1, \ldots, T$ has a unique parent denoted $\pi(n) \in \mathcal{N}_{t-1}$, and every node $n \in \mathcal{N}_{t}, t=0,1, \ldots, T-1$ has a nonempty set of child nodes $\mathcal{C}(n) \subset \mathcal{N}_{t+1}$. We denote the set of all nodes in the tree by $\mathcal{N}$. 
The set $\mathcal{A}(n)$ denotes the collection of ascendant nodes or path history of node $n$ including itself and $\mathcal{D}(n)$, the set of descendant nodes of $n$, again including itself. The probability distribution $P$ is obtained by attaching positive weights $p_{n}$ to each leaf node $n \in \mathcal{N}_{T}$ so that $\sum_{n \in \mathcal{N}_{T}} p_{n}=1$. For each non-leaf (intermediate level) node in the tree we have, recursively,

$$
p_{n}=\sum_{m \in \mathcal{C}(n)} p_{m}, \quad \forall n \in \mathcal{N}_{t}, t=T-1, \ldots, 0 .
$$

Hence, each non-leaf node has a probability mass equal to the combined mass of its child nodes.

A random variable $X$ is a real-valued function defined on $\Omega$. It can be lifted to the nodes of a partition $\mathcal{N}_{t}$ of $\Omega$ if each level set $\left\{X^{-1}(a): a \in \mathbb{R}\right\}$ is either the empty set or is a finite union of elements of the partition. In other words, $X$ can be lifted to $\mathcal{N}_{t}$ if it can be assigned a value on each node of $\mathcal{N}_{t}$ that is consistent with its definition on $\Omega$ [8]. This kind of random variable is said to be measurable with respect to the information contained in the nodes of $\mathcal{N}_{t}$. A stochastic process $\left\{X_{t}\right\}$ is a time-indexed collection of random variables such that each $X_{t}$ is measurable with respect $\mathcal{N}_{t}$. The expected value of $X_{t}$ is uniquely defined by the sum

$$
\mathbb{E}^{P}\left[X_{t}\right]:=\sum_{n \in \mathcal{N}_{t}} p_{n} X_{n}
$$

The conditional expectation of $X_{t+1}$ on $\mathcal{N}_{t}$ is given by the expression

$$
\mathbb{E}^{P}\left[X_{t+1} \mid \mathcal{N}_{t}\right]:=\sum_{m \in \mathcal{C}(n)} \frac{p_{m}}{p_{n}} X_{m} .
$$

The market consists of $J+1$ tradable securities indexed by $j=0,1, \ldots, J$ with prices at node $n$ given by the vector $S_{n}=\left(S_{n}^{0}, S_{n}^{1}, \ldots, S_{n}^{J}\right)$. We assume as in [9] that the security indexed by 0 has strictly positive prices at each node of the scenario tree. This asset corresponds to the risk-free asset in the classical valuation framework.

The number of shares of security $j$ held by the investor in state (node) $n \in \mathcal{N}_{t}$ is denoted $\theta_{n}^{j}$. Therefore, to each state $n \in \mathcal{N}_{t}$ is associated a vector $\theta_{n} \in \mathbb{R}^{J+1}$. The value of the portfolio at state $n$ is

$$
S_{n} \cdot \theta_{n}=\sum_{j=0}^{J} S_{n}^{j} \theta_{n}^{j}
$$

We need the following definition.

Definition 1 If there exists a probability measure $Q=\left\{q_{n}\right\}_{n \in \mathcal{N}_{T}}$ such that

$$
S_{t}=\mathbb{E}^{Q}\left[S_{t+1} \mid \mathcal{N}_{t}\right](t \leq T-1)
$$

then the vector process $\left\{S_{t}\right\}$ is called a vector-valued martingale under $Q$, and $Q$ is called a martingale probability measure for the process.

In our finite probability space setting an ACC $F$ generates payoff opportunities $F_{n},(n \geq 0)$ to its holder depending on the states $n$ of the market.

We use Figure 1 to illustrate the stochastic scenario tree. In this example there are only three periods. At the first period, which is denoted by $t=0$, stock prices are known, so there is only one node at this period. The index of this node is 0 . This node branches to three nodes at the second period. The three possible states for 


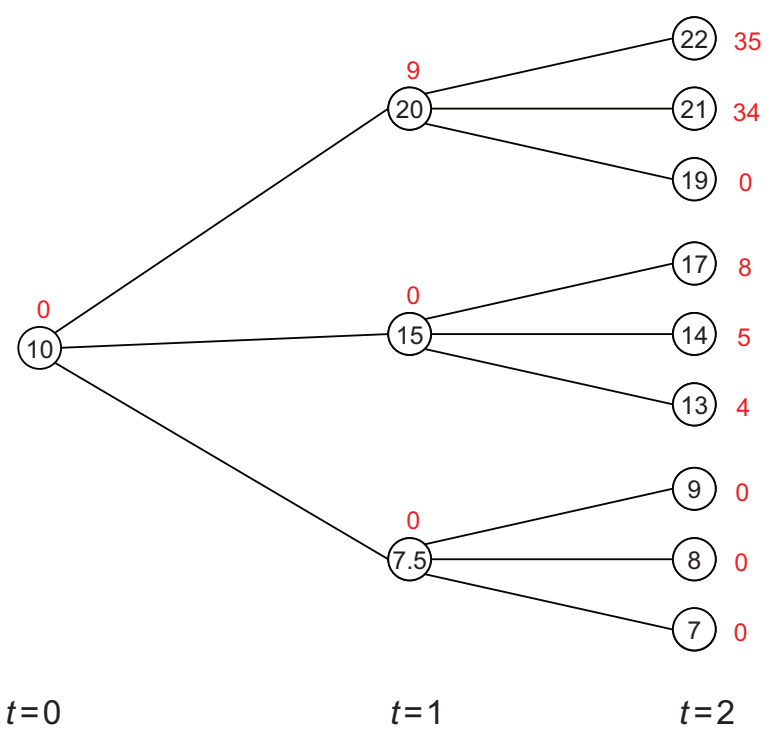

Figure 1. The tree representing the counterexample to the proof in [9].

the second period are respresented by three nodes: the upper node is indexed by 1 , the middle node is indexed by 2 and the lower node is indexed by 3 . Then, each node in the second period branches to three nodes at the third period. Hence, there are nine nodes at the third period. These nodes are indexed in the same fashion from 4 to 12. We assume that there are only two financial instruments in the market: a stock and a bond. Bond price is assumed to be 1 for each node, which means that the risk free interest rate is zero. The number inside each node represents the price of the stock at that node. The number next to a node represents the payoff of some fictitious contingent claim at that node. We will use this toy scenario tree and contingent claim as a counterexample below after the proof of Theorem 1 .

For further details on arbitrage-free pricing of ECC and ACC using stochastic linear programming we refer the reader to $[5,8,9]$.

\section{The main result}

We will now give a new proof of Theorem 3 of [9]. An arbitrage seeking buyer's problem can be formulated as the following problem that we will refer as AP1.

$$
\begin{aligned}
\max V & \\
\text { s.t. } \quad S_{0} \cdot \theta_{0} & =F_{0} e_{0}-V \\
S_{n} \cdot\left(\theta_{n}-\theta_{\pi(n)}\right) & =F_{n} e_{n}, \forall n \in \mathcal{N}_{t}, 1 \leq t \leq T \\
S_{n} \cdot \theta_{n} & \geq 0, \forall n \in \mathcal{N}_{T} \\
\sum_{m \in \mathcal{A}(n)} e_{m} & \leq 1, \forall n \in \mathcal{N}_{T} \\
e_{n} & \in\{0,1\}, \forall n \in \mathcal{N} .
\end{aligned}
$$

The optimal value of $V$ is the largest amount that a potential buyer is willing to disburse for acquiring a given ACC $F$. The computation of this quantity via 
the above integer programming problem is carried out by construction of a least costly (adapted) portfolio process replicating the proceeds from the contingent claim by self-financing transactions using the market-traded securities in such a way to avoid any terminal losses. The integer variables and related constraints represent the one-time exercise of the ACC; see [9] for further details.

A linear programming relaxation of AP1 is the following problem AP2:

$$
\max V
$$

s.t.

$$
\begin{aligned}
S_{0} \cdot \theta_{0} & =F_{0} e_{0}-V \\
S_{n} \cdot\left(\theta_{n}-\theta_{\pi(n)}\right) & =F_{n} e_{n}, \forall n \in \mathcal{N}_{t}, 1 \leq t \leq T \\
S_{n} \cdot \theta_{n} & \geq 0, \forall n \in \mathcal{N}_{T} \\
\sum_{m \in \mathcal{A}(n)} e_{m} & \leq 1, \forall n \in \mathcal{N}_{T} \\
e_{n} & \geq 0, \forall n \in \mathcal{N} .
\end{aligned}
$$

Theorem 1 There exists an optimal solution to AP2 with $e_{n} \in\{0,1\}, \forall n \in \mathcal{N}$.

Proof Assume that AP2 has an optimal solution $V^{*}, e^{*}$ and $\theta^{*}$ such that $e_{n}^{*} \notin\{0,1\}$ for some $n \in \mathcal{N}$.

Case 1 We will first consider the case where $e^{*}$ has a value not equal to 0 or 1 for the root, which is the starting node of the tree (i.e. $\left.e_{0}^{*} \notin\{0,1\}\right)$. In order to deal with this case, we will form the Lagrangian function for AP2. That is

$$
\begin{aligned}
L(V, e, \theta, x, y, z)= & V-y_{0}\left[S_{0} \cdot \theta_{0}-F_{0} e_{0}+V\right]-\sum_{n \in \mathcal{N} \backslash\{0\}} y_{n}\left[S_{n} \cdot\left(\theta_{n}-\theta_{\pi(n)}\right)-F_{n} e_{n}\right] \\
& +\sum_{n \in \mathcal{N}_{T}} x_{n} S_{n} \cdot \theta_{n}-\sum_{n \in \mathcal{N}_{T}} z_{n}\left[\sum_{m \in \mathcal{A}(n)} e_{m}-1\right] .
\end{aligned}
$$

After rearranging the above function we have

$$
\begin{aligned}
L(V, e, \theta, x, y, z)= & \left(1-y_{0}\right) V+\sum_{n \in \mathcal{N}_{T}}\left(x_{n}-y_{n}\right) S_{n} \cdot \theta_{n}+\sum_{n \in \mathcal{N} \backslash \mathcal{N}_{T}} \theta_{n} \cdot\left[\sum_{m \in \mathcal{C}(n)} y_{m} S_{m}-y_{n} S_{n}\right] \\
& +\sum_{n \in \mathcal{N}}\left[y_{n} F_{n}-\sum_{m \in \mathcal{D}(n) \cap \mathcal{N}_{T}} z_{m}\right] e_{n}+\sum_{n \in \mathcal{N}_{T}} z_{n} .
\end{aligned}
$$

Then the dual problem of AP2 can be formulated as

$$
\begin{aligned}
\min \sum_{n \in \mathcal{N}_{T}} z_{n} & \\
\text { s.t. } \quad y_{0} & =1 \\
{\left[x_{n}-y_{n}\right] S_{n} } & =0, \forall n \in \mathcal{N}_{T} \\
\sum_{m \in \mathcal{C}(n)} y_{m} S_{m} & =y_{n} S_{n}, \forall n \in \mathcal{N} \backslash \mathcal{N}_{T} \\
y_{n} F_{n}-\sum_{m \in \mathcal{D}(n) \cap \mathcal{N}_{T}} z_{m} & \leq 0, \forall n \in \mathcal{N} \\
x_{n}, z_{n} & \geq 0, \forall n \in \mathcal{N}_{T} .
\end{aligned}
$$


Since $S_{n} \neq 0$, second constraint implies that $x_{n}=y_{n}, \forall n \in \mathcal{N}_{T}$. Thus, the dual problem can be rearranged as

$$
\begin{aligned}
& \min \sum_{n \in \mathcal{N}_{T}} z_{n} \\
& \text { s.t. } \quad y_{0}=1 \\
& \sum_{m \in \mathcal{C}(n)} y_{m} S_{m}=y_{n} S_{n}, \forall n \in \mathcal{N} \backslash \mathcal{N}_{T} \\
& y_{n} F_{n}-\sum_{m \in \mathcal{D}(n) \cap \mathcal{N}_{T}} z_{m} \leq 0, \forall n \in \mathcal{N} \\
& y_{n}, z_{n} \geq 0, \forall n \in \mathcal{N}_{T} .
\end{aligned}
$$

We have an optimal solution to AP2 with $e_{0}^{*} \notin\{0,1\}$. Then complementary slackness implies that the third constraint of the above programme should be satisfied as an equality for the corresponding optimal solution of the dual problem (i.e. $y_{0} F_{0}-\sum_{m \in \mathcal{N}_{T}} z_{m}=0$ ). Since $y_{0}=1$, we have $F_{0}=\sum_{m \in \mathcal{N}_{T}} z_{m}$. Thus, the optimal solution to the dual problem is found to be $F_{0}$. Then, by strong duality we know that $F_{0}$ is the optimal value of AP2. One can easily show that a feasible solution to AP2 is $e_{0}=1, V=F_{0}$ and all the other variables as zeros (each $\theta_{n}$ as a zero vector) with objective value $F_{0}$. This is an optimal solution with $e_{n} \in\{0,1\}, \forall n \in \mathcal{N}$, thus the proof for the first case is complete.

Case 2 Now assume that optimal solution $e^{*}$ is such that $e_{0}^{*}=0$ and $e_{n}^{*} \notin\{0,1\}$ for some $n \in \mathcal{N}$. Let $I=\left\{i \mid e_{i}^{*} \notin\{0,1\}, i \in \mathcal{N}\right\}$. Let $G=\{g \mid g \in I, \mathcal{A}(g) \cap I=\{g\}\}$. Let $w$ be the element with the smallest time index (that is closest to the root) in $G$. Note that $e_{n}^{*}=0, \forall n \in \mathcal{A}(w) \backslash\{w\}$ in this case. Also, let $k$ denote the time index for node $w$.

Claim: One can always find an optimal solution to AP2 with $e_{w} \in\{0,1\}$ and $e_{i}=0$ for all $i \in \mathcal{A}(w) /\{w\}$.

To prove the claim we will consider the following two linear programmes to which we will refer as AR1 and AR2, respectively:

$$
\max e_{w}
$$

s.t. $S_{w} \cdot\left(\theta_{w}-\theta_{\pi(w)}^{*}\right)=F_{w} e_{w}$

$$
\begin{aligned}
S_{n} \cdot\left(\theta_{n}-\theta_{\pi(n)}\right) & =F_{n} e_{n}, \forall n \in \mathcal{D}(w) \backslash\{w\} \\
S_{n} \cdot \theta_{n} & \geq 0, \forall n \in \mathcal{N}_{T} \cap \mathcal{D}(w) \\
\sum_{m \in \mathcal{A}(n) \cap \mathcal{D}(w)} e_{m} & \leq 1, \forall n \in \mathcal{N}_{T} \cap \mathcal{D}(w) \\
e_{n} & \geq 0, \forall n \in \mathcal{D}(w),
\end{aligned}
$$

$\min e_{w}$

$$
\begin{aligned}
\text { s.t. } \quad S_{w} \cdot\left(\theta_{w}-\theta_{\pi(w)}^{*}\right) & =F_{w} e_{w} \\
S_{n} \cdot\left(\theta_{n}-\theta_{\pi(n)}\right) & =F_{n} e_{n}, \forall n \in \mathcal{D}(w) \backslash\{w\} \\
S_{n} \cdot \theta_{n} & \geq 0, \forall n \in \mathcal{N}_{T} \cap \mathcal{D}(w) \\
\sum_{m \in \mathcal{A}(n) \cap \mathcal{D}(w)} e_{m} & \leq 1, \forall n \in \mathcal{N}_{T} \cap \mathcal{D}(w) \\
e_{n} & \geq 0, \forall n \in \mathcal{D}(w) .
\end{aligned}
$$


Let us denote the optimal solution of AR1 as $\bar{\theta}_{\mathcal{D}(w)}, \bar{e}_{\mathcal{D}(w)}$ and to AR2 as $\tilde{\theta}_{\mathcal{D}(w)}, \tilde{e}_{\mathcal{D}(w)}$. If the optimal value of AR1 is 1 , then we see that $\left(\bar{\theta}_{\mathcal{D}(w)}, \theta_{\mathcal{N} \backslash \mathcal{D}(w)}^{*}\right),\left(\bar{e}_{\mathcal{D}(w)}, e_{\mathcal{N} \backslash \mathcal{D}(w)}^{*}\right)$ form another optimal solution of AP2 with $e_{w}=1$. For this optimal solution we have $e_{w}=1$ and $e_{i}=0, \forall i \in \mathcal{A}(w) \backslash\{w\}$ (we have also $e_{i}=0$, for all $i \in \mathcal{D}(w) \backslash\{w\}$ for this solution). Similarly, if the optimal value of AR2 is 0 , then $\left(\tilde{\theta}_{\mathcal{D}(w)}, \theta_{\mathcal{N} \backslash \mathcal{D}(w)}^{*}\right)$, $\left(\tilde{e}_{\mathcal{D}(w)}, e_{\mathcal{N} \backslash \mathcal{D}(w)}^{*}\right)$ form another optimal solution of AP2 with $e_{w}=0$. Then, for this optimal solution we have $e_{i}=0$, for all $i \in \mathcal{A}(w)$. So, our claim will be proved if we can show that AR2's having an optimal value greater than 0 implies that the optimal value of AR1 is 1 . To show that we will consider the dual problems of AR1 and AR2. The dual problems DAR1 and DAR2 of AR1 and AR2, respectively, are

$$
\begin{aligned}
\min \sum_{n \in \mathcal{N}_{T} \cap \mathcal{D}(w)} z_{n}+y_{w} S_{w} \cdot \theta_{\pi(w)}^{*} \\
\text { s.t. } \quad \sum_{m \in \mathcal{C}(n)} y_{m} S_{m}=y_{n} S_{n}, \forall n \in \mathcal{D}(w) \backslash \mathcal{N}_{T} \\
-y_{w} F_{w}+\sum_{n \in \mathcal{N}_{T} \cap \mathcal{D}(w)} z_{n} \geq 1 \\
y_{n} F_{n}-\sum_{m \in \mathcal{D}(n) \cap \mathcal{N}_{T}} z_{m} \leq 0, \forall n \in \mathcal{D}(w) \backslash\{w\} \\
\max -\sum_{n \in \mathcal{N}_{T} \cap \mathcal{D}(w)} z_{n}-y_{w} S_{w} \cdot \theta_{\pi(w)}^{*} \geq 0, \forall n \in \mathcal{N}_{T} \cap \mathcal{D}(w), \\
\text { s.t. } \sum_{m \in \mathcal{C}(n)} y_{m} S_{m}=y_{n} S_{n}, \forall n \in \mathcal{D}(w) \backslash \mathcal{N}_{T} \\
-y_{w} F_{w}+\sum_{n \in \mathcal{N}_{T} \cap \mathcal{D}(w)} z_{n} \geq-1 \\
y_{n} F_{n}-\sum_{m \in \mathcal{D}(n) \cap \mathcal{N}_{T}} z_{m} \leq 0, \forall n \in \mathcal{D}(w) \backslash\{w\} \\
y_{n}, z_{n} \geq 0, \forall n \in \mathcal{N} \mathcal{N}_{T} \cap \mathcal{D}(w) .
\end{aligned}
$$

We will denote the optimal value of AR2 by $\alpha$, which is equal to the optimal value of DAR2. We know that $\alpha \leq 1$. Assume that $\alpha>0$. Then by complementary slackness we know that the second constraint of DAR2 must be satisfied as an equality at the corresponding optimal solution, since $e_{w} \neq 0$ at the optimal solution of AR2. Then at the optimal solution of DAR2, we have

$$
0>\sum_{n \in \mathcal{N}_{T} \cap \mathcal{D}(w)} z_{n}+y_{w} S_{w} \cdot \theta_{\pi(w)}^{*} \geq-y_{w} F_{w}+\sum_{n \in \mathcal{N}_{T} \cap \mathcal{D}(w)} z_{n}=-1 .
$$

Moreover, we must have $y_{w} \geq 0$ for any feasible solution of DAR1 and DAR2. This follows from the following fact. We have $y_{n} \geq 0, \forall n \in \mathcal{N}_{T} \cap \mathcal{D}(w)$. Then, since $S_{n}^{0}>0$ for all $n$, we have $y_{n} \geq 0, \forall n \in \mathcal{N}_{T-1} \cap \mathcal{D}(w)$ by the first constraints of DAR1 and DAR2. Similarly, we can show the same successively for $(T-2),(T-3), \ldots, k$. So, we have $y_{w} \geq 0$. Then, using the second inequality of (1) 
we have

$$
\begin{aligned}
\sum_{n \in \mathcal{N}_{T} \cap \mathcal{D}(w)} z_{n}+y_{w} S_{w} \cdot \theta_{\pi(w)}^{*} & \geq-y_{w} F_{w}+\sum_{n \in \mathcal{N}_{T} \cap \mathcal{D}(w)} z_{n} \\
y_{w} S_{w} \cdot \theta_{\pi(w)}^{*} & \geq-y_{w} F_{w} \\
S_{w} \cdot \theta_{\pi(w)}^{*} & \geq-F_{w}
\end{aligned}
$$

where the last step follows from $y_{w} \geq 0$. Then, for DAR1 at any feasible solution we have

$$
1 \leq-y_{w} F_{w}+\sum_{n \in \mathcal{N}_{T} \cap \mathcal{D}(w)} z_{n} \leq y_{w} S_{w} \cdot \theta_{\pi(w)}^{*}+\sum_{n \in \mathcal{N}_{T} \cap \mathcal{D}(w)} z_{n}
$$

whence we see that the optimal solution of DAR1 cannot be less than 1 . It is easy to see by AR1 that optimal value of DAR1 cannot be greater than 1 either. Hence, we conclude that the optimal value of DAR1 and therefore that of AR1, is 1. This completes the proof of our claim. Using the claim we see that there always exists an optimal solution to AP2 with $e_{w} \in\{0,1\}$ and $e_{i}=0$ for all $i \in \mathcal{A}(w)$. So, one can eliminate all the nodes having time index $k$ in $I$ by applying the above procedure. Then, proceeding successively with the nodes in $(k+1)$ st, $(k+2)$ nd...(T)th time indices one can find an optimal solution for AP2 with $e_{n} \in\{0,1\}, \forall n \in \mathcal{N}$. We note that, at each step the size of $I$ might increase, but no nodes with a time index less than or equal to that of the node eliminated at that particular step can show up in $I$ at the next step. This completes the proof of the theorem.

In their proof Pennanen and King [9] claim that for an optimal solution of AP2 if the contingent claim is exercised partially at a node, then there is another optimal solution in which the contingent claim is fully exercised at that node. However, we have discovered counterexamples to this claim by computer experimentation. For some special cases, one can show, contrary to this claim, that there is another optimal solution where the claim is not exercised at that node, but no optimal solution exists in which the claim is fully exercised at that node.

For a counterexample to the claim of [9] let us return to the example of the fictitious contingent claim in Figure 1 at the end of Section 2. We wrote a simple GAMS code, which is illustrated in the appendix of this article, to construct and solve the buyer's problem (the linear programming relaxation of it) using CPLEX Version 9.0.2 with the data given in the example. The optimal value, i.e. the buyer's price, of this problem is 2. CPLEX 9.0.2 reports a fractional optimal solution of this problem where we have $e_{1}=0.625$. We show the non-zero variables of this solution in Table 1 . Here, $\theta_{n j}$ denotes the number of shares of security $j(j=0$ for the bond and

Table 1. The optimal values of variables in the counterexample (the remaining variables have value zero).

\begin{tabular}{lccccccccccccc}
\hline $\begin{array}{l}\text { Opt. } \\
\text { Value }\end{array}$ & $\theta_{00}$ & $\theta_{01}$ & $\theta_{10}$ & $\theta_{11}$ & $\theta_{20}$ & $\theta_{21}$ & $\theta_{50}$ & $e_{1}$ & $e_{4}$ & $e_{5}$ & $e_{7}$ & $e_{8}$ & $e_{9}$ \\
\hline 2 & 6 & -0.8 & 83.125 & -4.375 & 9 & -1 & 4.375 & 0.625 & 0.375 & 0.375 & 1 & 1 & 1 \\
\hline
\end{tabular}


$j=1$ for the stock) held by the investor at node $n$. Besides, $e_{n}$ is the variable for the execution time of the contingent claim.

If the proof in [9] were correct, according to their argument, we would have another optimal solution to this problem with $e_{1}=1$. However when we add the constraint $e_{1}=1$ and solve the same problem again, we see that the optimal solution becomes 1.8. This is contradicting the argument in [9]. While this example is based on a fictitious contingent claim, it illustrates the difficulty of defining an optimal 'rational' exercise policy. These difficulties are also discussed in [3]. In this example, it appears that the buyer could exercise early at node 1 , and take away 9 units since there is a possibility of not getting anything should the process end at node 6. However, such an early exercise is not optimal as the example shows. Such examples (one can find others that are similar) remain difficult to construct, but they clearly demonstrate the gap in the proof of [9].

Returning to the consequences of Theorem 1, this result shows that one can always find a feasible solution to AP1 that gives the optimal value of the relaxed problem AP2. Then, since the optimal value of a problem cannot be better than the optimal value of its relaxation we say that optimal value of AP1 can be found by solving AP2.

One major implication of this result is the passage to a linear programming problem from an NP-hard integer programming problem that is potentially very difficult to solve in practice. Linear programming algorithms with a computational complexity bounded above by a low-order polynomial in the number of variables and constraints for computing a solution to $\epsilon$-accuracy are well known and well studied; see Chapter 6 of [1]. For practical computation, the problem AP2 has $|\mathcal{N}|(J+2)+1$ variables and $|\mathcal{N}|+2\left|\mathcal{N}_{T}\right|$ constraints in addition to $|\mathcal{N}|$ non-negativity constraints. In practice, the state-of-the-art linear programming solvers can easily handle instances where the cardinality of $\mathcal{N}$ is 22,200 and the cardinality of $\mathcal{N}_{T}$ is $20,000[10]$.

A second implication is that one can use duality to get expressions for the buyer's price of the ACC in terms of martingale measures and stopping times as pointed out in the introduction. These aforementioned two results are given in [9]. Here we re-iterate the second major implication in detail, for the sake of completeness. For simplicity, we assume w.l.o.g. that $S_{n}^{0}=1, \forall n=1, \ldots, T$. We assume an interest-free environment. However, the more general case is easy to implement using the discounted price process of [8]. We will need the buyer's price of a ECC in order to find that of an ACC. The buyer's price of an ECC is derived in [8]. We will briefly show the derivation here. Under the assumption of an interest-free environment, the buyer's problem for an ECC with payoffs $F_{n}$ is

$$
\begin{aligned}
\max V & \\
\text { s.t. } \quad S_{0} \cdot \theta_{0} & =F_{0}-V \\
S_{n} \cdot\left(\theta_{n}-\theta_{\pi(n)}\right) & =F_{n}, \forall n \in \mathcal{N}_{t}, 1 \leq t \leq T \\
S_{n} \cdot \theta_{n} & \geq 0, \forall n \in \mathcal{N}_{T} .
\end{aligned}
$$


The dual problem of this programme is

$$
\begin{aligned}
& \min \sum_{n \in \mathcal{N}} y_{n} F_{n} \\
& \text { s.t. } \quad y_{0}=1 \\
& \sum_{m \in \mathcal{C}(n)} y_{m} S_{m}=y_{n} S_{n}, \forall n \in \mathcal{N} \backslash \mathcal{N}_{T} . \\
& y_{n} \geq 0, \forall n \in \mathcal{N}_{T} .
\end{aligned}
$$

Then, the buyer's price of an ECC can be expressed as

$$
\min _{Q \in \tilde{\mathcal{Q}}} \sum_{n \geq 0} q_{n} F_{n}
$$

where $\tilde{\mathcal{Q}}$ denotes the closure of the set of all martingale measures equivalent to $P$, i.e. the set

$$
\tilde{\mathcal{Q}}=\left\{q \mid q_{0}=1, q_{n} S_{n}=\sum_{m \in \mathcal{C}(n)} q_{m} S_{m}, \forall n \in \mathcal{N} \backslash \mathcal{N}_{T} ; 0 \leq q_{n}, \forall n \in \mathcal{N}_{T}\right\} .
$$

Define the sets

$$
\begin{gathered}
E=\left\{e \mid e \text { is }\left(\mathcal{F}_{t}\right)_{t=0}^{T} \text {-adapted, } \sum_{t=0}^{T} e_{t} \leq 1 \text { and } e_{t} \in\{0,1\} P \text {-a.s. }\right\}, \\
\tilde{E}=\left\{e \mid e \text { is }\left(\mathcal{F}_{t}\right)_{t=0}^{T} \text {-adapted, } \sum_{t=0}^{T} e_{t} \leq 1 \text { and } e_{t} \geq 0 P \text {-a.s. }\right\} .
\end{gathered}
$$

One common way to describe exercise strategies of ACCs is by stopping times. These are functions $\tau: \Omega \rightarrow\{0, \ldots, T\} \cup\{+\infty\}$ such that $\{\omega \in \Omega \mid \tau(\omega)=t\} \in \mathcal{F}_{t}$, for each $t=0, \ldots, T$. The relation $e_{t}=1 \Leftrightarrow \tau=t$ defines a one-to-one correspondence between stopping times and decision processes $e \in E$. The set of stopping times will be denoted by $\mathcal{T}$. The set $\tilde{E}$ corresponds to the set of randomized stopping times discussed extensively in [3].

THEOREM 2 ([9]) If there is no arbitrage in the market price process, the buyer's price for ACC F can be expressed as

$$
\max _{\tau \in \mathcal{T}} \min _{Q \in \tilde{Q}} \mathbb{E}^{Q}\left[F_{\tau}\right]=\min _{Q \in \tilde{Q}} \max _{\tau \in \mathcal{T}} \mathbb{E}^{q}\left[F_{\tau}\right] .
$$

Proof If we set $e$ fixed in AP1 and maximize with respect to $\theta$, we have a ECC with payoffs $F_{t} e_{t}$ for $t=0,1, \ldots, T$. Then, by (2), for the buyer's price of this ECC, we have

$$
\min _{Q \in \tilde{\mathcal{Q}}} \mathbb{E}^{Q}\left[\sum_{t=0}^{T} F_{t} e_{t}\right]
$$

Then, maximizing with respect to $e$, for the buyer's price of the ACC we have

$$
\max _{e \in E} \min _{Q \in \tilde{\mathcal{Q}}} \mathbb{E}^{Q}\left[\sum_{t=0}^{T} F_{t} e_{t}\right] \text {. }
$$


The correspondence between stopping times and the process $e \in E$ implies that the buyer' s price for the ACC can be expressed as the left-hand side of Equation (3) since maximization over $\mathcal{T}$ is equivalent to maximization over $E$ after making the appropriate change in the objective function. By Theorem 1, instead of last expression we can use

$$
\max _{e \in \tilde{E}} \min _{Q \in \tilde{\mathcal{Q}}} \mathbb{E}^{Q}\left[\sum_{t=0}^{T} F_{t} e_{t}\right] .
$$

Since $\tilde{E}$ and $\tilde{\mathcal{Q}}$ are bounded convex sets, by Corollary 37.6.1 of [11] we can change the order of max and min without changing the value. Then, for each fixed $Q \in \tilde{\mathcal{Q}}$, the objective in (4) is linear in $e$. So the maximum over $\tilde{E}$ is attained at an extreme point of $\tilde{E}$. We know that the extreme points of $\tilde{E}$ are the elements of the set $E$. Thus, we reach the expression on the right-hand side in (3).

We can extend our result for stocks that pay dividends or interest. We assume that there is no dividend associated with $S^{0}$. We have the following corollary (proven here for the first time, to the best of the authors' knowledge).

Corollary 1 If each security $j=1, \ldots, J$ pays dividend payments $D_{n}^{j}$ in node $n$, under the assumption of no arbitrage in the market price process, the buyer's price $F_{b}$ for an ACC F can be expressed as

$$
F_{b}=\max _{\tau \in \mathcal{T}} \min _{Q \in \tilde{\mathcal{Q}}^{\prime}} \mathbb{E}^{Q}\left[F_{\tau}\right]=\min _{Q \in \tilde{\mathcal{Q}}^{\prime}} \max _{\tau \in \mathcal{T}} \mathbb{E}^{q}\left[F_{\tau}\right]
$$

where

$$
\tilde{\mathcal{Q}}^{\prime}=\left\{q \mid q_{0}=1, q_{n} S_{n}=\sum_{m \in \mathcal{C}(n)} q_{m}\left(S_{m}+D_{m}\right), \forall n \in \mathcal{N} \backslash \mathcal{N}_{T} ; 0 \leq q_{n}, \forall n \in \mathcal{N}_{T}\right\} .
$$

Proof If dividends are paid, self-financing constraints of P1 becomes

$$
S_{n} \cdot\left(\theta_{n}-\theta_{\pi(n)}\right)-D_{n} \cdot \theta_{\pi(n)}=F_{n} e_{n}, \forall n \in \mathcal{N}_{t}, 1 \leq t \leq T .
$$

The rest of the argument, including the proof of Theorem 1 follows as it is in the case of stocks without dividends.

\section{Conclusions}

We presented an alternative proof of an interesting and important result announced by Pennanen and King [9] on the computation of the buyer's price of an ACC by linear programming instead of 0-1 integer programming. We included a GAMS code that helps illustrate some important arguments related to our proof. We also showed that the result is unaffected by dividend payments. While ECC prices were known to be computable using linear programming, the result opens the way to computing the prices of ACC also by linear programming, which allows the numerical solution of very large multi-period hedging problems.

The application of this approach to real market data is the subject of our future research. 


\section{Acknowledgement}

This research is partially supported by TUBITAK Grant 107K250 and a scholarship from the Fulbright Commission.

\section{References}

[1] A. Ben-Tal and A.S. Nemirovski, Lectures on Modern Convex Optimization: Analysis, Algorithms and Engineering Applications, MPS-SIAM Series on Optimization, SIAM, Philadelphia, 2001.

[2] A. Brooke, D. Kendrick, and A. Meeraus, GAMS: A User's Guide, The Scientific Press, San Fransisco, CA, 1992.

[3] P. Chalasani and S. Jha, Randomized stopping times and American option pricing with transaction costs, Math. Finan. 11 (2001), pp. 33-77.

[4] CPLEX Solver Manual, ILOG, 2007. http://www.gams.com/dd/das/solvers/cplex.pdf

[5] S.D. Flåm, Option pricing by mathematical programming, Tech. Rep. (2007) Available at: http://ideas.repec.org/p/hhs/lunewp/2007_010.html.

[6] H. Föllmer and A. Schied, Stochastic Finance: An Introduction in Discrete Time, De Gruyter Studies in Mathematics 27, 2nd ed., Walter de Gruyber, Berlin, 2004.

[7] J.M. Harrison and D.M. Kreps, Martingales and arbitrage in multiperiod securities markets, J. Econ. Theor. 20 (1979), pp. 381-408.

[8] A.J. King, Duality and martingales: A stochastic programming perspective on contingent claims, Math. Program. Ser. B 91 (2002), pp. 543-562.

[9] T. Pennanen and A. King, Arbitrage pricing of American contingent claims in incomplete markets - a convex optimization approach, Working paper, 2006 June 2006, Available at math.tkk.fi/ teemu/american.pdf.

[10] M.Ç. Pınar, Measures of model uncertainty and calibrated option bounds, Optimization 58 (2009), pp. 335-350.

[11] T.R. Rockafellar, Convex Analysis, Princeton University Press, Princeton, New Jersey, 1970.

[12] A. Shapiro, Stochastic programming approach to optimization under uncertainty, Math. Program. Ser. B 112 (2008), pp. 183-220.

\section{Appendix}

The GAMS code for the counterexample is as follows.

Variables z, theta00, theta01, theta10, theta11, theta20, theta 21 , theta 30 , theta 31 , theta 40 , theta41, theta50, theta51, theta60, theta61, theta70, theta71, theta80, theta81, theta90, theta91, theta100, theta101, theta110, theta111, theta120, theta121;

Positive Variables e0, e1, e2, e3, e4, e5, e6, e7, e8, e9, e10, e11, e12;

Equations node1, node2, node3, node4, node5, node6, node7, node8, node9, node10, node11, node12, noarb1, noarb2, noarb3, noarb4, noarb5, noarb6, noarb7, noarb8, noarb9, amer1, amer2, amer3, amer4, amer5, amer6, amer7, amer8, amer9, ob, extra;

ob.. $-\mathrm{z}=\mathrm{e}=$ theta $00+10 *$ theta $01-\mathrm{e} 0 * 0$;

node $1 . . \quad 0=\mathrm{e}=$ theta $10-$ theta $00+20 *($ theta $11-$ theta 01$)-\mathrm{e} 1 * 9$;

node $2 . . \quad 0=\mathrm{e}=$ theta 20 -theta $00+15 *($ theta $21-$ theta 01$)-\mathrm{e} 2 * 0$;

node $3 . . \quad 0=\mathrm{e}=$ theta $30-$ theta $00+7.5 *($ theta31-theta 01$)-\mathrm{e} 3 * 0$;

node $4 . . \quad 0=\mathrm{e}=$ theta $40-$ theta $10+22 *($ theta $41-$ theta 11$)-\mathrm{e} 4 * 35$;

node $5 . . \quad 0=\mathrm{e}=$ theta $50-\operatorname{theta} 10+21 *($ theta $51-$ theta 11$)-\mathrm{e} 5 * 35$; 


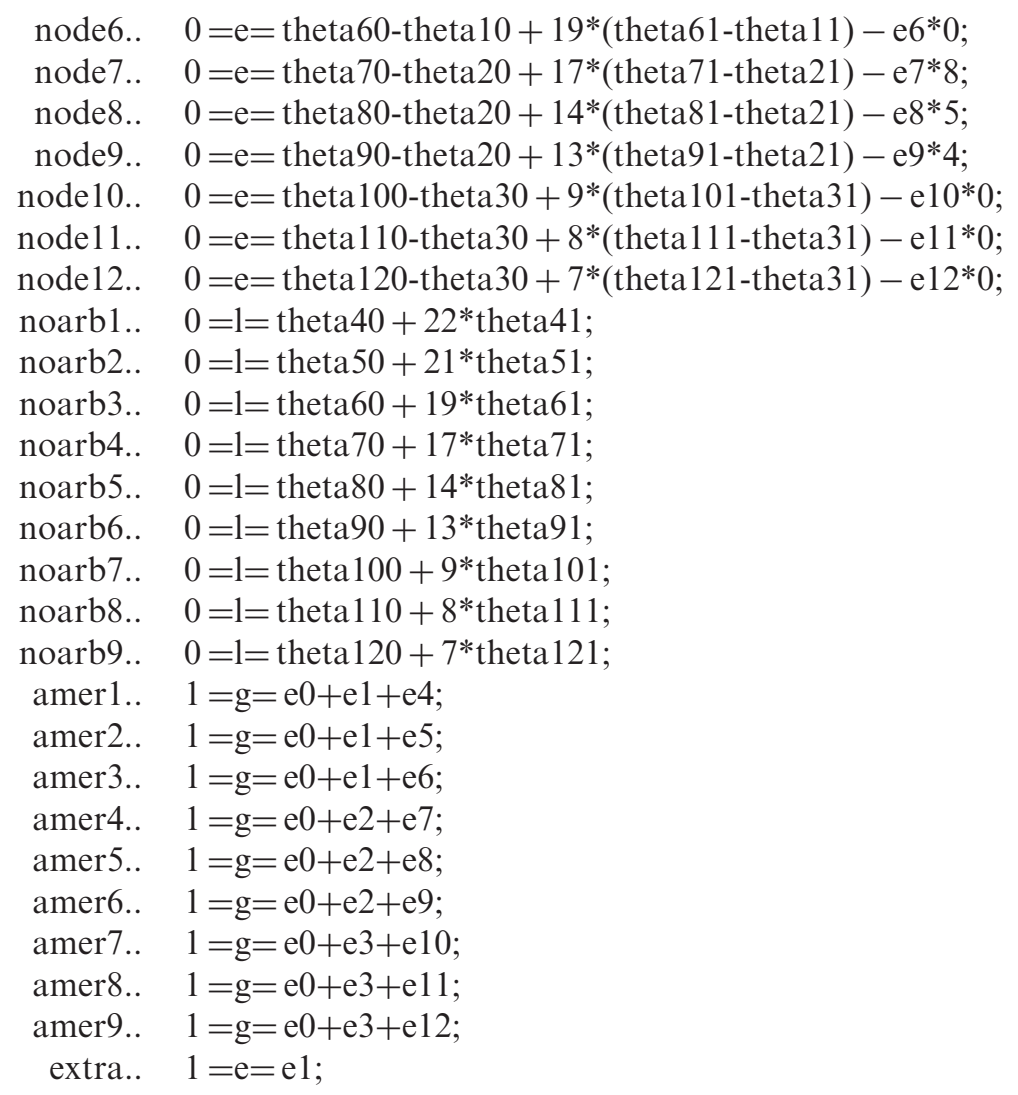

Model buyer /all/;

solve buyer using lp maximizing $\mathrm{z}$;

*The constraint named 'extra' is used to adjust the value of variable e1 for different values.

The rest of the code represents the model AP2 with values taken from the counterexample. 